\title{
TIME DOMAIN CHARACTERISTICS OF RF ENVIRONMENTS
}

\author{
Ming-Chiang Li and Gary T. Roan \\ Naval Research Laboratory, Washington D.C. 20375
}

Abstract

Radio frequency environments have conventionally been characterized by frequency domain techniques. The presentation made here takes a different approach and discusses time domain methods and various associated features of interest. It has been observed that these methods are useful not only for the simplicity with which they can characterize RF environments, but also for their application in electromagnetic compatibility testing.

\section{Introduction}

Many similarities exist between acoustical and radio frequency (RF) measurements. These similarities arise from their common range of wavelengths, although in the frequency spectrum the two are situated at almost opposite ends, audible sound at $20 \mathrm{~Hz}-20 \mathrm{KHz}$ and the radio band at $20 \mathrm{MHz}-20 \mathrm{GHz}$. This band of radio trequencies is the most highly trafficked, hosting numerous uses including communications, radar, remote surveillance, and electronic warfare.

Sound and RF sources are omnipresent, producing high levels of ambient noise which interfere with the intended signal, whether acoustic or electromagnetic. Often these sources are unpredictable and create severe problems. Interference can also come from undesirable multiple reflections along the path of propagation. All of these problems contribute to the difficulty of maintaining measurement integrity and lead to the high cost of acoustical and RF tests.

Many efforts have been made to overcome the above difficulties. Anechoic chambers, shielded enclosures, and mode stirring chambers were originally used to guard against unwanted interference during acoustic measurements. These enclosures have since been adapted for RF tests, providing an isolated environment from external source interference and thus ensuring measurement integrity.

Although above chambers remove the problems of external source interference, but cause more problems on the multipath interference. The environmental characteristics of acoustic chambers are well known. Multiple reflections of a sound wave in a total enclosure or partial enclosure create a series of distinct and overlapped echoes. Distinct echoes come from individual wells or objects in the enclosure. The overlapping acoustical echoes are referred to as the reverberation, which decays slowly and is most intrinsic to the acoustical environment. The investigation of the acoustical reverberation as initiated by Sabine ${ }^{1}$ at the turn of this century was a very important achievement in acoustics, and has lead us to a better understanding on the acoustical environments.

The description of RF environments has took a different road. RF chambers are described in terms of their response to each individual RF frequency. Similarities in the acoustical and RF phenomena lead us to believe that each important acoustical concept should have its own respective RF counterpart. Considering the intrinsic characteristics of the waves, one would naturally expect the occurrence of RF reverberation. The expectation can not be fulfilled. Except the use of a term "reverberation" to describe one type of particular RF chambers, the concept of reverberation is not generally employed in description of RF environments. The purpose of this presentation is to provide a discussion of such time domain RF characterizations.

\section{Short RE pulses}

When a continuous wave impinges on an object, the wave sets the object on a forced vibration. This common phenomenon can be divided into three different stages. These are the initial attack, steady state vibration, and final decay. The first and third stages are transient in nature depending heavily on how fast the continuous wave is formed and cut off. A slower wave forming and cutting off will mask the transient characteristics of the object. A short pulse has a better chance than a continuous wave in revealing the transient characteristics of the object, since it will not excite the steady state vibration.

A short RF pulse is usually created through a fast discharge process. The rate of discharge is determined by the resistance and capacitance of the discharge circuit. Large values of resistance and capacitance will slow the discharge processes and lead to stretched RF pulses. A fast RF discharge rate requires a small circuit and capacitance, which imposes an upper limit on the storage of electromagnetic energy and delivery of electromagnetic power. Short RF pulses typically lack power. Thus, while the short RF pulse may be suitable for probing the time domain characteristics of cables, it is useless for the RF characterization of open environments. Basically, discharge processes yield step pulses which are rich in low frequency RF components. The lowest frequency component of such step pulses is direct current (DC), and the highest component is determined by the pulse rise time. Since an antenna is required for radiating RF pulses, the alternating current $(A C)$ nature of bancwidth limited RF antennas will fitter out the low frequency contents of a step pulse. This filtering operation stretches the duration of the discharged pulse and further reduces its meager power.

The appearance of the coherent vector analyzer provides an alternative. The experimental information provided by a vector network analyzer is complete and can be transferred to the time domain to synthesize the measurements of powerful RF short pulses. The synthesized RF pulse width in a coherent measurement is the inverse of frequency band width. A vector network analyzer operates in a stepping mode and step through 8.0 GHz to $12.0 \mathrm{GHz}$ leading to a synthesized pulse with a quarter nanosecond width.

\section{Beverberation chamber}

The reverberation chamber is defined as an enclosure with a long reverberation time. The design objective of a reverberation chamber, aside from providing an isolated environment from the external source interferences, is to generate acoustically high level broad band fieids efficiently over a large test volume. The chamber utilizes the shielded, multimoded resonating environment with a high quality factor $Q$ to obtain uniformed fields with respect to the temporal, spatial, polarization, and directionality averages. This technique offers a time efficient, effective way to evaluate the acoustical power generated by a sound source. The RF counterpart of this acoustical reverberation chamber performs similar functions in addition to evaluating electromagnetic compatibility and vulnerability (EMC/EMV) performance of large electronic systems.

The chamber under the test is a $48^{\prime \prime} \times 58.25^{\prime \prime} \times 37.4^{\prime \prime}$ aluminum box with sheet thickness of $0.187^{\prime \prime}$. A Transco $9 \mathrm{C} 30900$ precision spiral antenna is placed inside the chamber and connected to the Port 1 of an HP 8510 network analyzer. An X-band standard horn is also placed inside the 


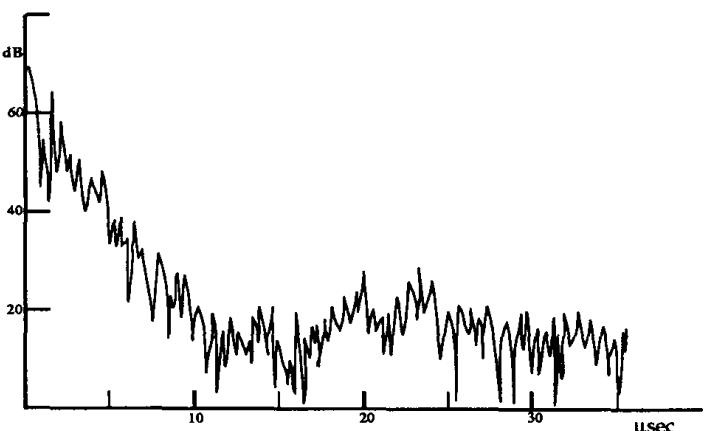

Fig. 1 Chamber reverberation $-S_{11}$ time dependance.

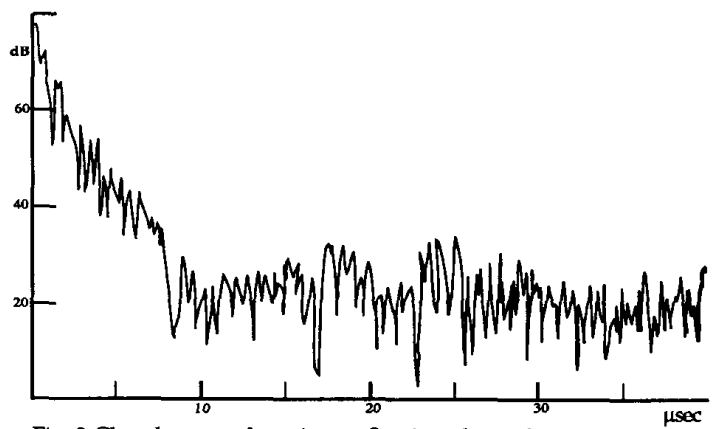

Fig. 2 Chamber reverberation $-S_{22}$ time dependance.

chamber and connected to the Port 2 of the same network analyzer. Figs. 1 and 2 depict the time dependances from frequency domain measurements. The starting frequency of $7.79 \mathrm{GHz}$ and stopping frequency of $7.89 \mathrm{GHz}$ are the same for both measurements. The width of synthesized pulse is 10 nanoseconds. The chamber in both cases is excited quickly and decays to its background ambience at about $60 \mathrm{~dB}$ below within $12 \mu \mathrm{sec}$ as seen in both Figs. 1 and 2 . Repeated

measurements have shown that the decay rate is independent of antenna positions and orientations in the chamber. According to the definition, the chamber reverberation is about $12 \mu \mathrm{sec}$. Figs. 3 and 4 depict the time dependances of the chamber RF characteristics as excited by real $10 \mu \mathrm{sec}$ rectangular pulses which center on the frequencies 12.227 and 12.599 $\mathrm{GHz}$ respectively. The dynamic range as shown in these two figures is only about $40 \mathrm{~dB}$ due to the weakness of the pulse strength. The excited chamber exhibits the common RF characteristics of enclosures. Three distinct stages - the initial attack, steady state, and final decay , are similar to those observed in the acoustics.

The reverberation time, as we have seen, provides a simple criterion for the characterization of an RF chamber. A longer reverberation time means more bounces of a trapped RF wave inside the chamber before it vanishes to zero. Each bounce spreads the RF power to different parts of the chamber and enhances the averaged RF field uniformity.

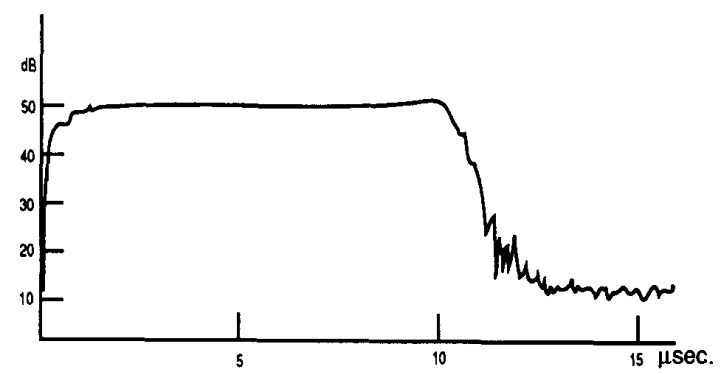

Fig. 3 The chamber is excited by a real pulse with frequency 12.227 $\mathrm{GHz}$ and pulse length $10 \mu \mathrm{sec}$.

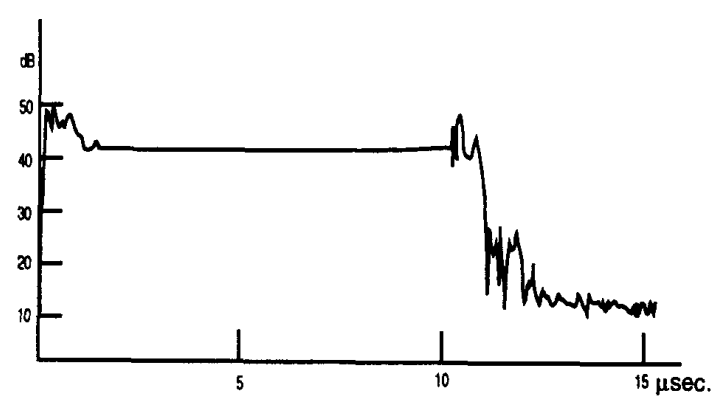

Fig. 4 The chamber is excited by a real pulse with frequency $12.599 \mathrm{GHz}$ and pulse length $10 \mu \mathrm{sec}$.

The reverberation time is an important measure to the quality of a reverberation chamber. A change in reverberation time means a change in the chamber characteristics. This parameter should be closely monitored during a test. The introduction of a test unit into the chamber will reduce the effective chamber volume and increase absorptive areas, hence altering the chamber characteristics. Close monitoring of the reverberation time is imperative. The altered reverberation time may serve as an effective indicator for the implementation of proper corrective measures in restoring the test integrity.

\section{Reverberatiuon Time}

The reverberation time $\mathrm{T}$ of an empty chamber in acoustics is predictable through the help of Sabine's equation

$$
T=K \frac{V}{\sum_{i} a_{i} A_{i}}
$$

where $K$ is a proportionality constant, $V$ the chamber volume, and $A_{j}$ the area of a wall element with an absorption coefficient $a_{i}$. The summation extends over all the distinct portions of the total surface area of the chamber. The above equation can be derived from the basic principles of wave theory. The similarity between the acoustical and RF waves leads us to believe that an equation equivalent to the above equation can also be derived from the basic electromagnetic theory for an RF chamber.

The above equation can lead to many interesting applications in the evaluation of RF absorption of different materials. The reverberation time of a reverberation chamber depends on the absorption of the wall elements. Placing an absorptive sample on a wall element will alter the reverberation time of the reverberant compartment. The observed reverberation times in Figs. 3 and $\mathbf{4}$ are shorter than that in Figs. 1 and 2. It is because a small RF absorptive material was inserted inside the chamber for the measurements of Figs. 3 and 4 . The RF field inside a high quality reverberation chamber is nearly uniform and isotropic, and will not be easily modified by edge diffractions of the sample. This provides a method for measuring the sample absorption.

\section{Acknowledgement}

Authors would like to thank Mr. Williams Lenzi for his support of this project, and to Mr. Mike Hatfield for interesting discussions.

1. W. C. Sabine,"Collected Papers on Acostics," Dover, New York, pp 103 (1964); L. L. Beranek," The notebooks of Wallace C. Sabine," J. Acost. Soc. Am., 61, pp. 629-639 (1977). 